\title{
ERRATUM
}

Horm. metabol. Res. 20 (1988) 1-10

(C) Georg Thieme Verlag Stuttgart . New York

\section{Mutant Insulin Syndromes}

\section{A. Vinik and G. Bell}

Departments of Medicine and Surgery. The University of Michigan, Ann Arbor, and The Howard Hughes Medical Institute, and the Departments of Biochemistry and Molecular Biology and Medicine, The University of Chicago, Chicago, Illinois, U.S.A.

In this article we inadvertently published a wrong Figure 5 . The correct Figure 5 is as follows:

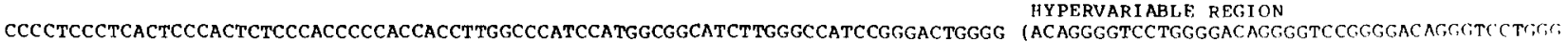

GACAGGGGTGTGGGGACAGGGGTCTGGGGACAGGGGTGTGGGGACAGGGGTGTGGGGACAGGGGTCTGGGGACAGGGGTGTGGGGACAGGGGTCCGGGGACAGGGGTGTGGGGACAGGGGT CTGGGGACAGGGGTGTGGGGACAGGGGTGTGGGGACAGGGGTCTGGGGACAGGGGTGTGGGGACAGGGGTCCTGGGGACAGGGGTGTGGGGACAGGGGTGTGGGGACAGGGGTGTGGGGAC AGGGGTGTGGGGACAGGGGTCCTGGGGATAGGGGTGTGGGGACAGGGGTGTGGGGACAGGGGTCCCGGGGACAGGGGTGTGGGGACAGGGGTGTGGGGACAGGGGTCCTGGGGACAGGGGT CTGAGGACAGGgGTGTGGGCACAGGGGTCCTGGGGACAGGGGTCCTGGGGACAGGGGTCCTGGGGACAGGGGTCTGGGG) ACAGCAGCGCAAAGAGCCCCGCCCTGCAGCCTCCAGCTCTCC TGGTCTAATGTGGAAAGTGGCCCAGGTGAGGGCTTTGCTCTCCTGGAGACATTTGCCCCCAGCTGTGAGCAGGGACAGGTCTGGCCACCGGGCCCCTGGTTAAGACTCTAATGACCCGCTG GTCCTGAGGAAGAGGTGCTGACGACCAAGGAGATCTTCCCACAGACCCAGCACCAGGGAAATGGTCCGGAATTGCAGCCTCAGCCCCCAGCCATCTGCCGACCCCCCCACCCCAGGCCCT EXON 1

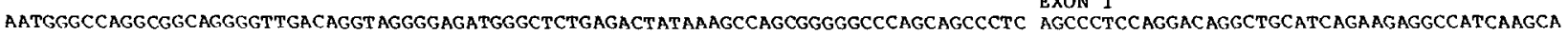
INTRON A

G GTCTGTTCCAAGGGCCTTTGCGTCAGGTGGGCTCAGGGTTCCAGGGTGGCTGGACCCCAGGCCCCAGCTCTGCAGCAGGGAGGACGTGGCTGGGCTCGTGAAGCATGTGGGGTGAGCCC

Ala Leu Leu Ala Leu Trp Gly Pro Asp Pro Ala Ala Ala Phe Val Asn Gln His Leu Cys Gly Ser Hís Leu Val Glu Ala Leu Tyr Leu GCG CTG CTG GCC CTC TGG GGA CCT GAC CCA GCC GCA GCC TTT GTG AAC CAA CAC CTG TGC GGC TCA CAC CTG GTG GAA GCT CTC TAC CTA

\section{0}

30

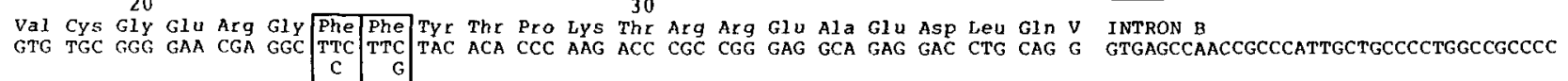

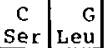

CAGCCACCCCCTGCTCCTGGCGCTCCCACCCAGCATGGGCAGAAGGGGGCAGGAGGCTGCCACCCAGCAGGGGGTCAGGTGCACTTTTTTAAAAAGAAGTTCTCTTGGTCACGTCCTAAAA GTGACCAGCTCCCTGTGGCCCAGTCAGAATCTCAGCCTGAGGACGGTGTTGGCTTCGGCAGCCCCGAGATACATCAGAGGGTGGGCACGCTCCTCCCTCCACTCGCCCCTCAAACAAATGC CCCGCAGCCCATTTCTCCACCCTCATTTGATGACCGCAGATTCAAGTGTTTTGTTAAGTAAAGTCCTGGGTGACCTGGGGTCACAGGGTGCCCCACGCTGCCTGCCTCTGGGCGAACACCC CATCACGCCCGGAGGAGGGCGTGGCTGCCTGCCTGAGTGGGCCAGACCCCTGTCGCCAGCCTCACGGCAGCTCCATAGTCAGGAGATGGGGAAGATGCTGGGGACAGGCCCTGGGGAGAAG TACTGGGATCACCTGTTCAGGCTCCCACTGTGACGCTGCCCCGGGGCGGGGGAAGGAGGTGGGACATGTGGGCGTTGGGGCCTGTAGGTCCACACCCAGTGTGGGTGACCCTCCCTCTAAC CTGGGTCCAGCCCGGCTGGAGATGGGTGGGAGTGCGACCTAGGGCTGGCGGGCAGGCGGGCACTGTGTCTCCCTGACTGTGTCCTCCTGTGTCCCTCTGCCTCGCCGCTGTTCCGGAACCT

$$
\text { EXON } 3
$$

al GIy Gln Val Glu Leu Gly Gly Gly Pro Gly Ala Gly Ser Leu Gin Pro Leu Ala Leu Glu Gly Ser Leu GCTCTGCGCGGCACGTCCTGGCAG TG GGG CAG GTG GAG CTG GGC GGG GGC CCT GGT GCA GGC AGC CTG CAG CCC TTG GCC CTG GAG GGG TCC CTG Gln Lys Arg Gly Ile Val Glu Glin Cys Cys Thr Ser Ile Cys Ser Leu Tyr Gln Leu Glu Asn Tyr Cys Asn AM

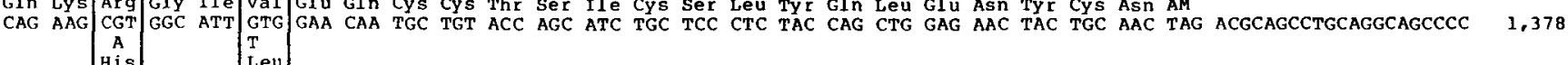
POLYADENYLATION SITE

Fig. 5 The sequence of the insulin gene, including the hypervariable region, the enhancer/promotor region, the exons encoding the insulin B-chain, C-peptide, the A chain, and the two introns which are present in the primary transcript of the insulin gene, but must be removed before the insulin messenger RNA can be translated. Also shown in boxes are the substitutions in the insulin gene that lead to aminoacid replacements. 